\title{
Characterizing Slow Photochemical Reaction Kinetics by Enhanced Sampling of Rare Events with Capillary Optical Fibers and Kramers' Theory
}

René A. Nome, ${ }^{*}{ }^{\dagger}$ Amanda F. Costa, ${ }^{\dagger}$ Jessica Lepkoski, $^{\dagger, \S}$ Gabriel A. Monteiro, ${ }^{\dagger}$ Juliano G. Hayashi, ${ }^{\ddagger, \|}$ and Cristiano M. B. Cordeiro

${ }^{\dagger}$ Institute of Chemistry, State University of Campinas, Campinas, SP 13083-970, Brazil

${ }^{\ddagger}$ Institute of Physics Gleb Wataghin, State University of Campinas, Campinas, SP 13083-859, Brazil

Supporting Information

ABSTRACT: Characterization of slow chemical reactions is essential for assessing catalytic efficiency in chemistry and biology. Traditionally, chemical reaction rates are obtained from population relaxation kinetics measurements and the Arrhenius equation. Unfortunately, it is difficult to use this approach to characterize reactions wherein concentrations change slowly. Thus, it is interesting to see whether a dynamical view of chemical reactions may be used to obtain the reaction rates of slow processes. In the present work, we

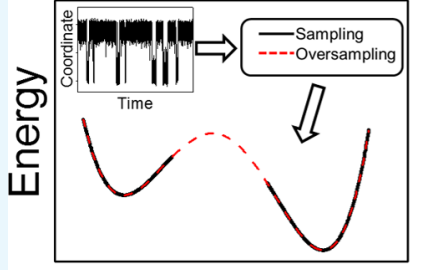

Reaction coordinate

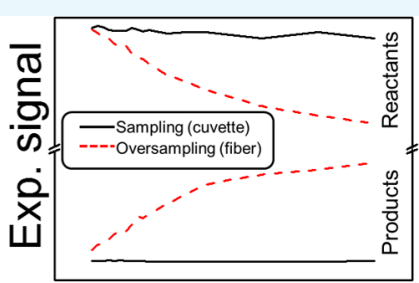

Time perform Brownian dynamics simulations of an asymmetric double-well potential to investigate how enhanced sampling of barrier crossing at transition states improves the characterization of reaction rate constants. We then present the design of a liquid-filled capillary optical fiber-based fluorescence spectrometer, which, like rare events, is also based on Poissonian statistics. We use the instrument to characterize the slow photochemical degradation kinetics of poly[2-methoxy-5-(2-ethylhexyloxy)-1,4-phenylenevinylene] (MEH-PPV) in $o$-dichlorobenzene. We have employed in situ optical microscopy measurements and electrodynamics simulations to characterize the excitation beam profile inside a liquid-filled capillary fiber. We compare the cuvette and capillary fiber sample holders and show that the MEH-PPV fluorescence line shape is independent of the sample holder, as expected. We characterize the photochemical degradation kinetics of MEH-PPV in $o$-dichlorobenzene solutions placed in the cuvette versus that in the capillary fiber. We observe small and slow changes in the time-dependent fluorescence spectra when the degradation reaction is performed in the cuvette. On the other hand, we are able to characterize reactant-concentration decay and product-concentration buildup from the time-dependent fluorescence spectra recorded during photochemical degradation of MEH-PPV performed inside the capillary optical fiber. Ultrafast optically heterodyne-detected optical Kerr effect spectroscopy and multimode Brownian oscillator analysis provide further insights into the role of bath oscillator modes of friction in the mechanism of MEH-PPV photochemical degradation. Overall, the work presented herein shows that slow photochemical degradation kinetics of MEH-PPV can be successfully and efficiently assessed in the capillary fiber fluorescence spectrometer.

\section{INTRODUCTION}

Characterizing the long-time kinetics of molecular events is an interesting problem that has received considerable attention over the past few years. For instance, in bimolecular reactions, which are of fundamental importance in chemistry, three rare (Poissonian) events must occur simultaneously for successful detection by spectroscopic methods: both reactants must diffuse through the optically probed volume, and there must be a reactive encounter between them within that volume. ${ }^{1,2}$ Slow reactions rates are especially challenging in this regard because very few barrier-crossing events occur per unit time. Initial-rate methods are commonly employed to estimate slow first-order rates, whereas no such simple method is currently available to characterize slow second-order reactions.

Experimental characterization of slow kinetics is of fundamental importance in basic and applied research. For example, an understanding of enzymatic activity ${ }^{3}$ requires experimental characterization of both catalyzed and uncatalyzed reaction rate constants. Although many enzymatic reactions occur on the microsecond to millisecond time scale, the corresponding uncatalyzed reactions are estimated to exhibit half-life values ranging from hours to billions of years. Similarly, organic polymers employed in solar cells and light-emitting diodes face similar challenges for the understanding of active material photodegradation mechanisms. ${ }^{4}$ Thus, it would be desirable to design new tools that probe all of the reactant molecules all of the time. Although there have been many approaches designed to detect single molecules in solution, on

Received: January 3, 2017

Accepted: May 17, 2017

Published: June 16, 2017 

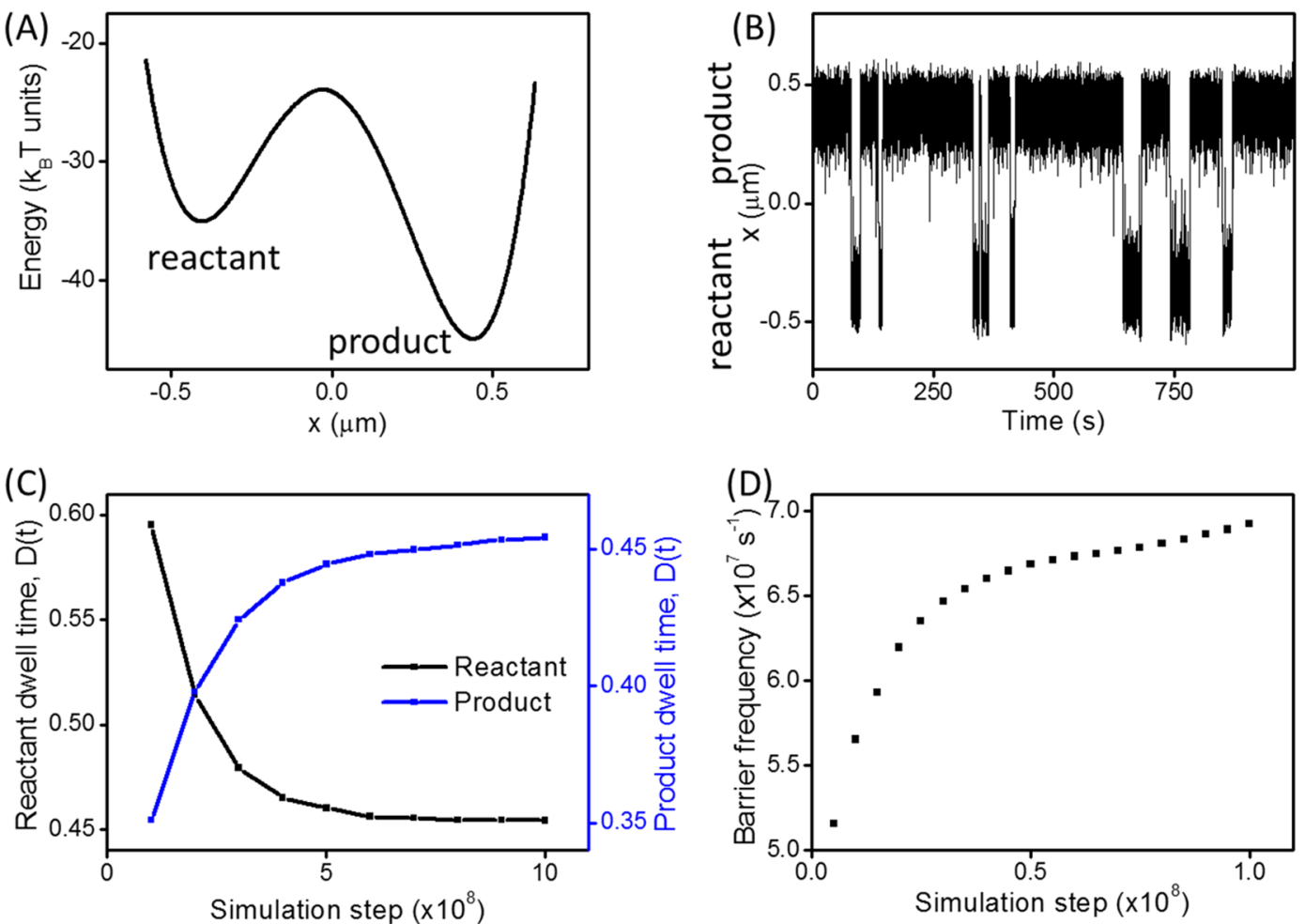

Figure 1. Langevin dynamics simulations in an asymmetric double well. (A) Energy landscape as a function reaction coordinates used in the simulations; (B) sample particle position time series trajectory obtained from the numerical simulations; (C) reactant and product dwell-time distributions as a function of time; (D) Kramers' theory barrier frequency as a function of simulation time. Simulation parameters: time step $=10^{-3} \mathrm{~s}$; product/reactant coordinates at $\pm 0.5 \mu \mathrm{m}$; particle size $R=1 \mu \mathrm{m} ; \eta=10^{-3} \mathrm{~Pa}$ s.

surfaces, or under confinement, ${ }^{5-14}$ characterization of the entire sample with single-molecule techniques in solution has not been reported so far.

The present work describes the design of a liquid-filled capillary optical fiber-based fluorescence spectrometer and its application in the characterization of the slow photochemical degradation kinetics of poly[2-methoxy-5-(2-ethylhexyloxy)1,4-phenylenevinylene] (MEH-PPV) in o-dichlorobenzene. We have employed in situ optical microscopy measurements and electrodynamics simulations to characterize the excitation beam profile inside the liquid-filled capillary fiber. Next, we compare the cuvette and capillary fiber sample holders and show that the MEH-PPV fluorescence line shapes are independent of the sample holder. Finally, we characterize the photochemical degradation kinetics of MEH-PPV in o-dichlorobenzene solutions placed in the cuvette versus that in the capillary fiber. Small changes in the time-dependent fluorescence spectra are observed during the photochemical reaction in the cuvette. On the other hand, we characterize both reactant-concentration decay and product-concentration buildup from the timedependent fluorescence spectra during photochemical degradation of MEH-PPV inside the capillary optical fiber. Overall, the work presented herein shows that the slow photochemical degradation kinetics of MEH-PPV can be successfully and efficiently characterized by simply inserting the solution of interest into the capillary fiber fluorescence spectrometer.

\section{RESULTS AND DISCUSSION}

To gain insights into why enhanced sampling of chemical kinetics may improve characterization of reaction rates, we have numerically solved the Langevin equation of motion (eqs 1 and 2) for an overdamped Brownian particle in an asymmetric double-well potential separated by a barrier (Figure 1A). The potential shown in Figure 1A is perhaps the simplest chemical reaction model that can be used to calculate reaction rates from a dynamical simulation (eqs 3 and 4). In our experimental results, shown in Figure 3, we study the photochemical polymer degradation kinetics, so it is important to consider why a twostate model can be used to study systems with multiple degrees of freedom, such as polymers. In the Supporting Information, we describe a polymer model with multiple configurations and energies in terms of a free-energy function that maps onto the two-state model shown in Figure 1A, and then, we present a mathematical model that can used to describe both rugged and simple two-state energy landscapes on the same footing. Thus, we believe that enhanced sampling of reaction rates can be useful for describing reaction rates not only for the model shown in Figure 1A but also as a useful reduced description of models with three or more states.

Figure 1B shows a typical particle position time series trajectory obtained by solving eq 1 . Given the stochastic nature of the simulations, each simulation run will yield a different trajectory. Nonetheless, overall, the trajectories exhibit features that depend on the specific parameters used to describe the energy landscape. For spontaneous reactions at equilibrium, described by the energy landscape shown in Figure 1A, the system spends most its time in three regions: the reactant and product wells and the barrier-crossing region. The trajectories are characterized by longer dwell times in the product state, and the number of barrier-crossing events per unit time decreases for slower reactions compared to that for faster reactions. Within this context, for a given chemical reaction, enhanced sampling improves determination of dwell and transition times, which are used to calculate reaction rates from transition state 

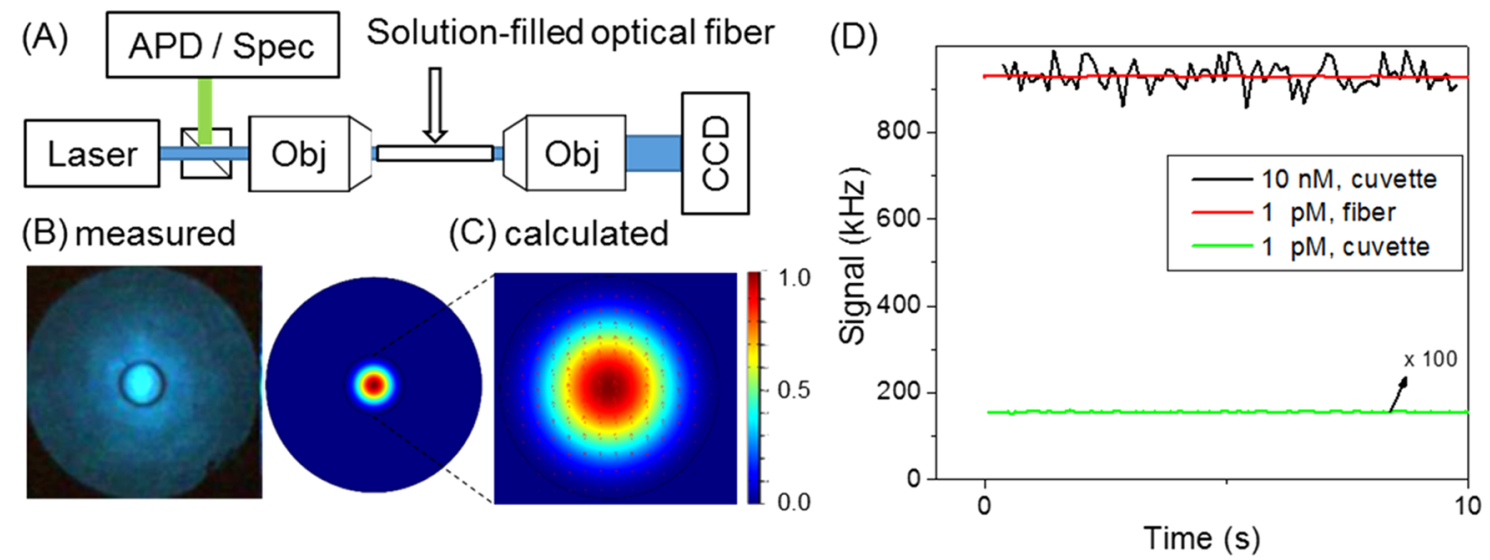

Figure 2. Liquid-filled capillary optical fiber fluorescence spectrometer. (A) Layout of the setup employed for the capillary optical fiber apparatus. APD: single-photon-counting detector; Spec: spectrometer; Obj: objective. (B) Experimentally measured spatial profile along the capillary fiber. (C) Calculated energy fluxes along the optical fiber axis for the capillary optical fiber system employed in the present work. Simulation parameters: external diameter $=125 \mu \mathrm{m}$; internal diameter $=25 \mu \mathrm{m}$; excitation wavelength $\lambda=470 \mathrm{~nm} ; n_{\text {DMSO }}=1.478$. (D) Photon-counting rate as a function of time for fluorescein in dimethyl sulfoxide (DMSO): $10 \mathrm{nM}$ solution in the cuvette (black curve), $1 \mathrm{pM}$ solution in the cuvette (green), and $1 \mathrm{pM}$ inside the capillary optical fiber (red).
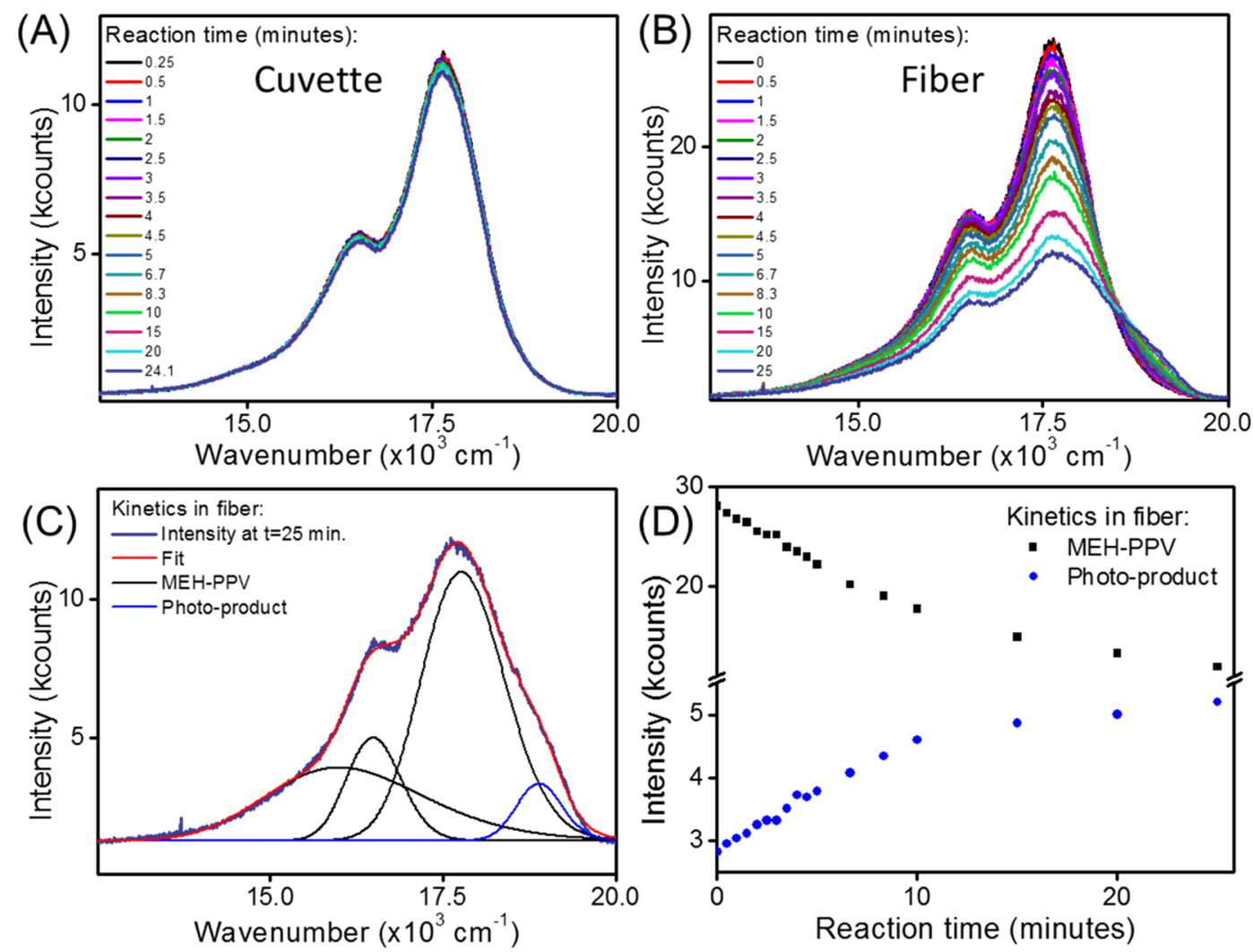

Figure 3. Photochemical degradation of MEH-PPV in o-dichlorobenzene. Time-dependent fluorescence spectra of the MEH-PPV/odichlorobenzene solution placed (A) in the cuvette and (B) inside the capillary fiber. (C) Franck-Condon analysis of the fluorescence spectrum of MEH-PPV/o-dichlorobenzene at $t=1500 \mathrm{~s}$. (D) Photoluminescence intensity as a function of time for MEH-PPV (black squares) and the MEHPPV photoproduct (blue circles). MEH-PPV concentration: $1 \times 10^{-5}$ monomer mol L ${ }^{-1}$.

theory and Kramers' theory, respectively. Additionally, we note that dwell and transition times are related to well and barrier frequencies, respectively, in an energy-landscape perspective. We study these two perspectives in Figure 1C,D.

Figure $1 \mathrm{C}$ shows the dwell-time distributions for reactant and product states as a function of simulation time from our numerical simulation results. To calculate the curves shown in Figure 1C, we first sort the reactant and product state trajectory segments from individual trajectories. Dwell-time distributions, calculated using eq 5 , give the fraction of molecules that occupy each state as a function of time. Because the reactant and product distributions fit well to single-exponential functions, we find that individual trajectories obey the two-state kinetics governed by the energy landscape shown in Figure 1A. Overall, the results shown in Figure 1C demonstrate that analysis of averaged individual trajectories can be used to extract 
information about population relaxation kinetics. Thus, we can use our numerical results to investigate how reaction rate determination can be improved by enhanced sampling of individual particle trajectories.

We have also used particle position probability distributions obtained numerically to extract potential energy curves and reaction rates from eqs 3 and 4, respectively. Thus, Figure 1D shows Kramers' theory barrier frequencies as a function of simulation time. Our approach thus provides a simple assessment of how enhanced sampling of thermally activated barrier crossing may be used to determine reaction rates. When we fitted reactant and barrier wells early on in the simulation, the resulting parameters converged to the equilibrium result as the simulation time increased. That is, the well frequency converges faster, which is consistent with the fact that the particle remains in the reactant well during a large fraction of the simulation time, especially for higher activation energies and slower reaction kinetics. As the number of transitions increases, the barrier frequency also converges quickly, presumably due to its linear dependence on the rate. As a result, we achieve an upper bound for the reaction rate well before the activation energy has converged to the value consistent with the underlying Boltzmann probability distribution.

Overall, the dynamical simulation results shown in Figure 1 demonstrate how enhanced sampling of rare events improves reaction rate characterization. We note that single-molecule fluorescence spectroscopy has been used recently to investigate barrier-crossing dynamics. ${ }^{11}$ The experiments reported in ref 11 probed time series trajectories of individual states and state-tostate barrier-crossing events; data analysis showed how to extract both transition state theory and Kramers' theory rate constants from the measured time series. On the other hand, the main point of the present work was to improve reaction rate constant determination during slow kinetic processes employing typical reactant concentrations (e.g., millimolar). Thus, we aim at designing a simple apparatus capable of comparing sampling approaches without necessarily resorting to single-molecule techniques. Poissonian statistics governs the dynamics of both (i) rare events in chemical kinetics and (ii) reactant diffusion through the excitation volume in photochemistry and photophysics. In the simulations shown in Figure 1 , we have increased the sampling of rare events by decreasing the simulation time step. In our experiments shown in Figures 2 and 3, we increase the sampling of rare events by replacing the sample holder from the cuvette to a liquid-filled capillary fiber such that the excitation volume becomes the entire sample volume. Figure $2 \mathrm{~A}$ shows the apparatus used in the present work; we have performed single-molecule detection (Figure 2D) as well as simpler detection schemes (Figure 3).

As shown in Figure 2A, the apparatus presented in this work is essentially a horizontal fluorescence microspectrometer employing a liquid-filled capillary optical fiber as the sample holder. Capillary optical fibers can be filled with any liquid/ solution of interest; waveguiding throughout the capillary is achieved as long as the refractive index of the core liquid/ solution is higher than that of the cladding. As a result, the excitation volume in solution-filled capillary optical fibers is the same as the entire sample volume, enabling more efficient fluorescent spectroscopy measurements. The capillary fiber sample holder also reduces the background and out-of-focus fluorescence when compared to those of the cuvette sample holder, thereby also improving the signal-to-noise ratio. On the other hand, the fluorescence cone associated with emission angles smaller than the critical angles for total internal reflection inside the fiber limits the total detected intensity. Overall, the solution-filled capillary fiber is expected to enable spectroscopic and kinetic measurements by fluorescence spectroscopy with improved sensitivity. Related sample holders, whereby the solution of interest flows through a hollow-core optical fiber, have been described previously in the context of bulk solution fluorescence detection ${ }^{15-18}$ and photochemical kinetics. ${ }^{19-21}$ However, we note that in the present work we do not use flowing solutions and our sample volume is the same as the entire observation volume, as shown in Figure 2A.

Figure 2B shows an optical microscopy image of the transverse spatial profile of light transmitted through a liquidfilled capillary optical fiber. Given the fiber length of $10 \mathrm{~cm}$, the image shown in Figure $2 \mathrm{~B}$ corresponds to the average transverse spatial profile integrated over the longitudinal propagation direction. Figure $2 \mathrm{~B}$ shows light intensity predominantly at the fiber core, with negligible light in the cladding, thereby confirming waveguide action. The complex beam profile in the cladding suggests that higher-order modes also couple to the capillary fiber nucleus, consistent our with electrodynamics simulation results (see below). Replacement of the solvent-filled capillary fiber with a MEH-PPV solution-filled capillary fiber leads to similar beam spatial profiles (Supporting Information).

Figure 2C shows the calculated contour plots of the transverse spatial distribution of the electric-field intensities inside the liquid-filled capillary optical fiber. The electrodynamics numerical simulations employed experimental parameters to model light-matter interactions: fiber external diameter, $125 \mu \mathrm{m}$; fiber internal diameter, $25 \mu \mathrm{m}$; silica cladding refractive index, 1.458; liquid core refractive index, 1.478 (DMSO), ${ }^{22}$ and incident light field wavelength, $470 \mathrm{~nm}$. The simulation results shown in Figure $2 \mathrm{C}$ indicate that the fundamental mode inside the optical fiber exhibits a Gaussian intensity distribution, consistent with direct imaging of the beam profile inside the fiber shown in Figure 2B. Similar conclusions were reached when we tested other solvents as the core pure liquid: toluene $(n=1.496)$ and $o$-dichlorobenzene $(n$ $=1.551){ }^{22}$ Thus, single-mode coupling to the fiber is believed to be predominant under the current experimental conditions because we employ a short, mechanically unstrained fiber excited by a plane wave. Nonetheless, the simulations also show that higher-order modes are supported as well (Supporting Information), exhibiting a more complex, refractive indexdependent transverse spatial profile. The fundamental mode is the most confined and thus has the largest effective index among all modes, whereas higher-order modes have smaller refractive indexes, thus leading to light spatial intensities in the cladding as well.

Focusing laser light with objectives typically employed in fluorescence microscopy gives approximately $1 \mathrm{fL}$ excitation volumes. $^{23}$ By contrast, the excitation volume in the present solution-filled capillary optical fiber is the same as the entire sample volume. In the present work, we used a $25 \times$ objective and a capillary fiber with a $12.5 \mu \mathrm{m}$ inner radius and $10 \mathrm{~cm}$ length; thus, our sample/excitation volume is approximately 10 $\mathrm{nL}$, which is 10000 times higher than the excitation volume commonly employed in single-molecule or bulk fluorescence microscopy instruments. To test the advantages and limitations of the capillary optical fiber design for characterization of slow reaction kinetics, in principle, any sample can be employed. In 
the present work, we have tested two systems: fluorescein in DMSO was used to verify the gain in sensitivity (Figure 2D), whereas MEH-PPV in o-dichlorobenzene (Figure 3) was used to compare reaction kinetics in the fiber versus the cuvette.

We have measured the single-photon count rate as a function of time for $10 \mathrm{nM}$ and $1 \mathrm{pM}$ solutions of fluorescein in DMSO (Figure 2D). The count rate measurements were performed both in the cuvette (black and green lines in Figure 1D) and inside the fiber (red line in Figure 1D) so that a direct comparison can be made. Solution concentrations of $10 \mathrm{nM}$ are commonly employed in single-molecule fluorescence correlation spectroscopy, whereas $1 \mathrm{pM}$ concentrations are more challenging. As shown by the black curve in Figure 1D, the 10 $\mathrm{nM}$ solution placed in a cuvette yielded an average of 950 kcounts $\mathrm{s}^{-1}$. On the other hand, the $1 \mathrm{pM}$ solution gave a signal of approximately 1 kcounts $\mathrm{s}^{-1}$, close to the background noise level of our apparatus (green curve in Figure 1D). The red curve in Figure $1 \mathrm{D}$ is the count rate as a function of time measured when placing the $1 \mathrm{pM}$ solution inside the optical fiber. Interestingly, the count rate was nearly $950 \mathrm{kcounts}^{-1}$, which is similar to the signal level obtained with the $1 \mathrm{nM}$ fluorescein solution placed in the cuvette. Thus, we observe a gain in sensitivity of approximately 1000 in going from the 1 pM solution in the cuvette to the $1 \mathrm{pM}$ solution in the capillary fiber, presumably due to the larger excitation volume afforded by placing the sample of interest inside the optical fiber. Despite signal intensity losses due to the fluorescence cone associated with emission angles smaller than the critical angles for total internal reflection in the capillary optical fiber, we still observe a significant gain in sensitivity afforded by probing the entire sample volume with a liquid-filled capillary fiber.

We note that this simple technique may also be applied to bimolecular reactions other than photochemical processes and that spectroscopic techniques other than fluorescence may be used as well, such as transmission/absorption spectroscopy in the visible or infrared regions. Nonetheless, we emphasize that the main point of this article is to study the connection between enhanced sampling and the characterization of chemical reaction rates. In connection with the simulation results presented in Figure 1, for a given observation time, sampling a larger portion of the trajectory at a higher rate yields more information for reaction rate determination. Likewise, Figure 2 describes a simple apparatus that is capable of extracting more data (emitted photons) in the same amount of time when compared to similar setups employing cuvette sample holders. The aforementioned alternative techniques would also benefit from enhanced sampling to characterize reaction rates.

To test whether the apparatus shown in Figure 2 can be useful in the characterization of slow chemical reaction kinetics, we have chosen to study the photochemical degradation of MEH-PPV. Photodegradation of MEH-PPV involves largely the reaction of the polymer with singlet oxygen, generated by sensitization involving the conjugated polymer triplet state. We note that photochemical degradation of MEH-PPV is a complex yet extensively studied reaction. However, we emphasize that the purpose of the kinetic studies shown in Figure 3 is to assess how enhanced sampling can help in the characterization of reaction rates, in connection with our results presented in Figures 1 and 2.

Figure 3 shows the time- and frequency-resolved photochemical degradation of a solution of MEH-PPV in odichlorobenzene. At time zero, we observe very good agreement between the MEH-PPV solution fluorescence line shapes obtained in the cuvette and capillary fiber sample holders (Supporting Information). Both spectra are consistent with previously reported MEH-PPV emission spectra ${ }^{24}$ and will be further discussed below. We have compared the timeresolved fluorescence spectra of MEH-PPV in $o$-dichlorobenzene in the cuvette and placed inside the capillary fiber, respectively (Figure 3A,B). Inside the capillary fiber, we have observed time-dependent changes in both the reactant and product concentrations during photochemical degradation of MEH-PPV solution, as shown in Figure 3B. Specifically, three spectral features characterize MEH-PPV photochemical degradation in the capillary fiber: (i) increase in emission intensity centered at $524 \mathrm{~nm}$ as a function of time, (ii) decrease in the integrated intensity associated with unreacted MEH-PPV emission, and (iii) an isosbestic point observed at $538 \mathrm{~nm}$. As shown in Figure 3A, in the control experiment performed in a cuvette while otherwise keeping the same experimental conditions as those in Figure 3B, we observe only a small decrease in emission intensity after $\sim 25 \mathrm{~min}$ of photochemical reaction time.

We quantify MEH-PPV photochemical degradation kinetics in the cuvette versus that in the capillary fiber. We employ the same incident light power and reaction time conditions so that a direct comparison of cuvette versus capillary fiber can be made. MEH-PPV photochemical degradation clearly proceeds at a much faster rate inside the capillary fiber (Figure 3B) when compared to that of the reaction performed in the cuvette (Figure 3A) under the same experimental conditions as those in Figure 3A. Bimolecular photochemical kinetics is dependent on the number of light-matter interaction processes and on collisions among reactants. Inside the capillary fiber, we excite the entire sample volume, thereby, in principle, sampling all reactive encounters.

Spectroscopic analysis of the spectra shown in Figure $3 \mathrm{~A}$ clearly reveals that we need an additional Gaussian line shape centered at $524 \mathrm{~nm}$ to adequately fit the MEH-PPV emission spectrum once the photochemical degradation reaction has begun (Figure 3C). The blue-shifted emission spectra of the degradation product relative to the MEH-PPV $0-0$ emission band suggests a light-induced decrease in the polymer conjugation length, which lowers the exciton diffusion length and increases electronic energy-level spacing, as discussed previously. ${ }^{24}$ Thus, we assign the emission peaks centered at 524 and $560 \mathrm{~nm}$ to the photodegradation product and unreacted MEH-PPV, respectively. Our results reported in Figure 3B,C are consistent with the spectroscopic changes described in previous reports on the photochemical oxidation of MEH-PPV and structurally related PPV derivatives. During oxidation, the increasing concentration of carbonyl groups and concomitant decrease in the number of double bonds both lead to a decrease in polymer conjugation length, thereby resulting in the observed blueshift and fluorescence quenching shown in Figure 3B,C. ${ }^{25-29}$ Full analysis of the time-dependent line shapes is consistent with this interpretation (see below).

Figure 3D shows the emission intensity as a function of reaction time for unreacted MEH-PPV (black points) and the photochemical degradation product (blue circles), where we have assigned both species in Figure 3C. Both increasing photoproduct intensity and decreasing MEH-PPV intensity occur as a function of reaction time, and after nearly $25 \mathrm{~min}$, both the reactant and product emission intensities asymptotically reach equilibrium. Although photochemical MEH-PPV degradation is observed when the reaction is performed in the 

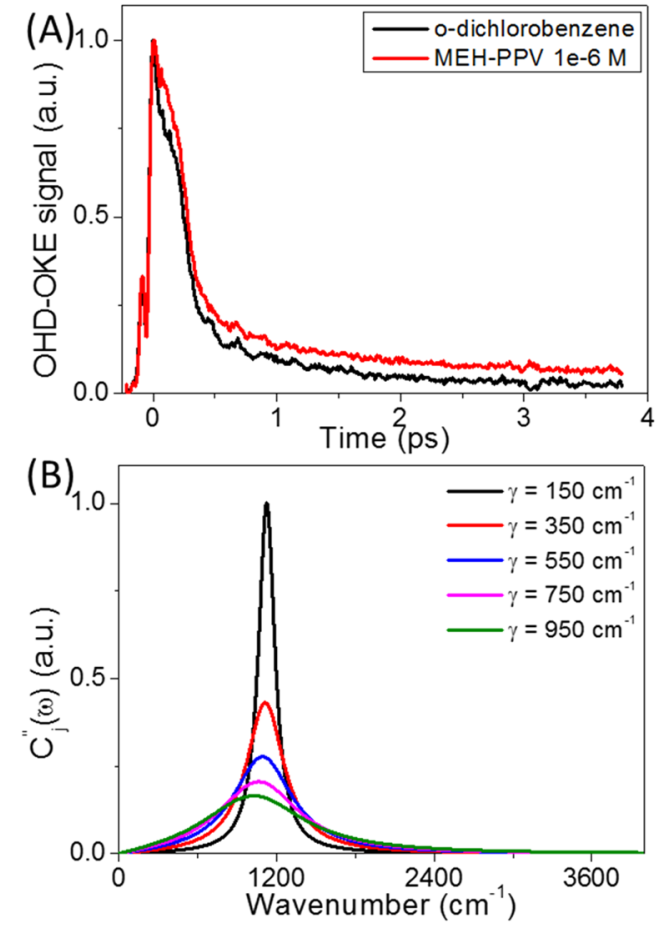

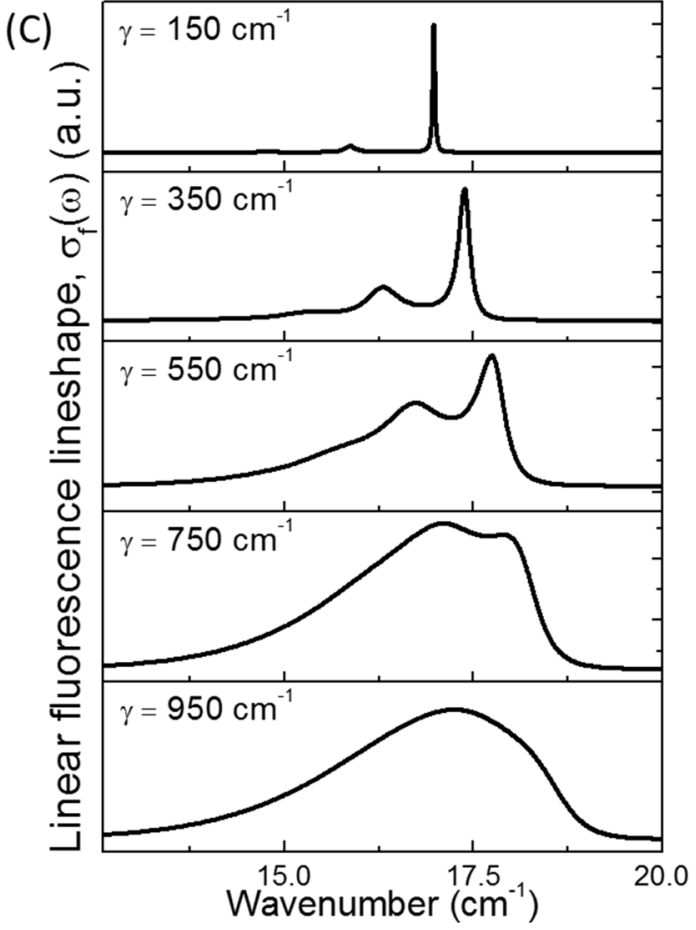

Figure 4. (A) Ultrafast OHD-OKE dynamics of MEH-PPV in $o$-dichlorobenzene (red curve) and pure solvent (black curve). (B) Spectral densities for the MBO model with $\omega_{j}=1126 \mathrm{~cm}^{-1}$ and $d_{j}=4$. The solid lines correspond to different values of damping (in units of $\mathrm{cm}^{-1}$ ), $\gamma_{j}: 150(\mathrm{black}), 350$ (red), 550 (blue), 750 (pink), and 950 (green). (C) Linear fluorescence line shapes calculated from the Brownian oscillator Hamiltonian using the same spectral densities and parameters as those in (B). $T=300 \mathrm{~K}$.

cuvette (Figure 3A), the corresponding reactant and product kinetics is much slower. The MEH-PPV photochemical degradation reaction times reported previously range from 5 min to several hours, depending on both sample preparation and illumination conditions. ${ }^{25-29}$

Overall, the results presented in Figure 3 show that it is possible to extract more useful information during the same amount of time using the capillary fiber sample holder when compared with the cuvette sample holder. Thus, the results presented in Figure 3 are consistent with the simulations presented in Figure 1. Although such an advantage in the collection efficiency of capillary fibers over the sample holder had been reported previously, ${ }^{14-21}$ to the best of our knowledge, this is the first demonstration of the capillary fiber design improving the chemical reaction rate characterization by enhanced sampling of rare events.

In the present work, we use ultrafast spectroscopy to parameterize (constrain) time-domain correlation functions, which are then used to calculate line shape functions and steady-state fluorescence spectra via the multimode Brownian oscillator (MBO) model. Thus, to further investigate the line shape changes shown in Figure 3B, we employ the correlation function formalism of molecular spectroscopy to extract frequency-domain information (frequencies and linewidths) from time-domain measurements and simulations. ${ }^{30}$ Although ultrafast coherent Raman spectroscopy is a time-domain thirdorder nonlinear laser spectroscopy technique, it has found widespread application in the characterization of steady-state vibrational resonances in chemistry and biology. ${ }^{31,32}$ Similarly, relaxation rates measured directly in the time domain parameterize correlation function-based line shape functions used to calculate steady-state fluorescence spectra. ${ }^{30}$ Thus, within the context of the present work aimed at investigating oversampling in chemical kinetics, we assess whether measuring the full spectra (Figure $3 \mathrm{~A}-\mathrm{C}$ ) rather than population-level changes (Figure 3D) can add to our understanding of the nature of system-bath interactions in this reaction.

We have performed ultrafast optically heterodyne-detected optical Kerr effect (OHD-OKE) spectroscopy measurements. OHD-OKE spectroscopy is an off-resonant transient birefringence technique that measures the polarizability correlation function. Thus, this technique can also be used to obtain lowfrequency spectral densities. ${ }^{33}$ Moreover, OHD-OKE spectroscopy is most sensitive to solvent nuclear motions; thus, differences in the OHD-OKE decay profiles between the solvent and solution were interpreted as being due to soluteinduced changes in the bath spectral density. Importantly, the bath spectral density retrieved from OHD-OKE can be used to calculate fluorescence line shapes using the $\mathrm{MBO}$ model that incorporates coupling of nuclear motions to optical transitions. That is, ultrafast OHD-OKE measurements are formally related to fluorescence line shapes by way of the correlation function formalism of molecular spectroscopy. ${ }^{30}$

Our ultrafast OHD-OKE spectroscopy measurements for $o$ dichlorobenzene as well as 1E-6M MEH-PPV dissolved in chlorobenzene are shown in Figure 4A. The time profiles shown in Figure 4A are typical of ultrafast OHD-OKE spectroscopy of simple chlorobenzene derivatives. ${ }^{34}$ At time zero, the instantaneous liquid response is associated with electronic nonlinear polarizability. This main peak is followed by beats and overdamped relaxation arising from coherently driven librational motion ( $<150 \mathrm{fs})$, "interaction-induced" intermolecular response $(\sim 200-600 \mathrm{fs})$, and diffusive reorientation $(>1 \mathrm{ps})$. The data shown in Figure $4 \mathrm{~A}$ was fitted by convolution of the instrument response function with the material response employing nonlinear least-squares fitting. 
The fit parameters for the transients shown in Figure 4A are summarized in the Supporting Information. The OHD-OKE signal of the MEH-PPV/o-dichlorobenzene solution also exhibits a prominent contribution from the electronic hyperpolarizability near time zero and a librational motion decay time ( $80 \mathrm{fs}$ ) similar to that of pure $o$-dichlorobenzene. However, the remaining decay components and corresponding amplitudes differ from those of the pure solvent. In particular, the interaction-induced decay time for the solution $(250 \mathrm{fs})$ is faster than that for the pure solvent (500 fs). Similarly, diffusive reorientation also decreases for the solution (1 ps) compared to that for the pure solvent ( $6 \mathrm{ps})$. The faster response times in solution compared to those in the solvent indicate stronger solvent-induced damping of solute nuclear modes.

To further assess solute-solvent coupling, we calculate timecorrelation functions and spectral densities for a single-mode Brownian oscillator as a function of damping frequency. On the basis of the results presented in Figures 3 and 4, we employ a single oscillator at $1180 \mathrm{~cm}^{-1}$ and vary the bath oscillator frequency from 150 to $950 \mathrm{~cm}^{-1}$. The spectral densities and fluorescence spectra are shown in Figure 4B,C, respectively. As expected, the modeled time-correlation functions decay faster with increasing bath frequency; this trend is also consistent with the ultrafast OHD-OKE data shown in Figure 4A. Accordingly, the spectral densities obtained by Fourier transformation of the calculated time-correlation functions shown in Figure 4B indicate stronger coupling of the solute to the solvent degrees of freedom over a larger spectral range as the bath frequency increases. Likewise, we have calculated the fluorescence line shapes shown in Figure 4C employing the MBO model with the same line shape function parameters and spectral densities as those shown in Figure 4B. As the damping frequency increases from 150 to $950 \mathrm{~cm}^{-1}$, the line shapes change from Lorentzian curves with clear vibronic coupling to broad and featureless Gaussian curves. We note that the fluorescence line shape calculated with damping frequency $=550 \mathrm{~cm}^{-1}$ is similar to the starting fluorescence spectra of $\mathrm{MEH}-\mathrm{PPV} / \mathrm{o}$-dichlorobenzene solutions. Furthermore, by further increasing the bath frequency from 550 to $950 \mathrm{~cm}^{-1}$, we note that the calculated fluorescence line shape exhibits both spectral broadening and blueshift. Thus, the line shapes calculated with the MBO model bear qualitative resemblance with the time-dependent fluorescence spectra shown in Figure 3.

Overall, the results shown in Figure 4 show how ultrafast spectroscopy and $\mathrm{MBO}$ calculations can be useful for investigating processes occurring in slower timescales (e.g., as shown in Figure 3A). The formal connection between ultrafast time-domain OKE spectroscopy and frequency-domain fluorescence is well-known. ${ }^{30}$ Nonetheless, the time-domain correlation function formalism of molecular spectroscopy is normally employed to study dynamics. In the present work, we explore the ability of ultrafast OKE spectroscopy to measure frequency-domain spectral densities, which, with the MBO model, are used in the calculation of full fluorescence line shapes. Our investigation of line-broadening mechanisms presented in Figure 4 shows how the system-bath interaction strength parameter obtained from ultrafast spectroscopy measurements and simulations (Figure 4A,B) is manifested in the calculated fluorescence spectra. We note that the connection between time-dependent line-broadening mechanisms shown in Figure 3C and the ultrafast spectroscopy/MBO model has not been described previously.
In summary, we have investigated how enhanced sampling may be used to study chemical kinetics. Brownian dynamics simulations of an asymmetric double well showed how oversampling enables characterization of rate constants on the basis of transition state and Kramers' theory, thereby leading to a more accurate characterization of chemical reaction rates. A liquid-filled capillary optical fiber-based fluorescence spectrometer is used to characterize the photochemical degradation kinetics of MEH-PPV in o-dichlorobenzene. Only small changes in the time-dependent fluorescence spectra are observed when the degradation reaction is performed in the cuvette (control experiment), whereas photochemical MEHPPV degradation kinetics was characterized when the degradation reaction was performed inside the capillary optical fiber. In addition to population-level changes, the fluorescence line shape was investigated employing the correlation function formalism of molecular spectroscopy. Whereas enhanced sampling has been shown previously to provide further insights into single-molecule dynamics, chemistry, and biology, ${ }^{11,35,36}$ the work presented herein illustrates how it can also be of interest in chemical and photochemical kinetics.

\section{METHODS}

Liquid-Filled Capillary Fiber Fabrication and Characterization. The capillary fiber was fabricated from an ultrapure silica tube (Heraeus) using a fiber-drawing tower. The electric current flowing through a graphite nucleus controls the drawing temperature, which in the present work was held constant at $1950{ }^{\circ} \mathrm{C}$. We control the internal and external diameters of the capillary fiber (25 and $125 \mu \mathrm{m}$, respectively) during fabrication through (i) a preformed tuning speed inside the oven, (ii) the fiber-drawing speed, and (iii) the nitrogen pressure applied inside the tube. During fiber drawing, we add the acrylate polymer to the external side of the fiber to improve its mechanical resistance.

To fill the capillary fiber with the liquid of interest, we insert one of the fiber ends into a syringe needle. The fiber was then cleaved and glued to the syringe needle. Each capillary fiber was individually inspected with a fiber optic microscope (Thorlabs) to assess cleavage quality. Liquid insertion into the fiber nucleus was then performed by pressurizing the syringe filled with the solution of interest. The capillary fibers thus prepared were placed in the optical setup for spectroscopy and microscopy experiments (Figure 2). Each liquid-filled capillary fiber was used only once and disposed of afterward, and a freshly prepared liquid-filled capillary fiber was employed for each experiment.

Light propagation inside the capillary fiber was characterized experimentally and compared with electrodynamics simulations employing the finite element method performed using COMSOL Multiphysics modeling software. ${ }^{37}$ Optical alignment inside the capillary fiber was investigated by observing the capillary fiber (i) side-on and (ii) end-on. In approach (i), topview images of the capillary fiber were obtained by employing a conventional upright microscope with a $10 \times$ objective and a digital camera (Paxcam 5); the images were analyzed quantitatively with Image.$^{38}$ In approach (ii), we first focus the incident laser light at the fiber/air interface. Then, the objective is moved along the optical axis with a micrometerresolution linear translation stage until a two-dimensional Gaussian spatial beam profile centered at the fiber nucleus is observed. End-on images were obtained by sending light output from the fiber end to a CCD camera coupled to a $60 \times$ objective 
(Laser Beam Profiler, Newport). The alignment procedure just described was used in all measurements described in the present work. All measurements were performed at $21{ }^{\circ} \mathrm{C}$ and $50 \%$ relative humidity.

Fluorescence Spectroscopy Inside Hollow-Core Liquid-Filled Capillary Optical Fibers. A $470 \mathrm{~nm}$ laser (PicoQuant) operating in the CW mode is sent to the capillary optical fiber employing a pair of mirrors and a dichroic mirror coupled with a color filter (Chroma). Laser intensity was controlled with a variable neutral density filter wheel, and the results shown in the present work employed $20 \mu \mathrm{W}$ incident light power on the sample. A beam splitter sends a small portion of the excitation light to a single-photon avalanche photodiode (Perkin Elmer) to monitor alignment and laser intensity fluctuations. Light coupling into the capillary optical fiber is performed with a three-axis platform (Thorlabs) equipped with $25 \times$ microscope objective (Newport). The liquid-filled capillary optical fiber is mounted on the platform employing a V-Groove fiber holder and magnetic clamps. Emission from the sample injected into the capillary optical fiber is collected in backward-fluorescence geometry and sent to a single-photon avalanche photodiode (Perkin Elmer) or spectrometer (Ocean Optics) for photon-counting and photoluminescence spectral measurements, respectively. Throughout the experiments described below, transmitted light was sent to a photodiode (Thorlabs) and CCD (Newport) for throughput and beam-profiling measurements, respectively. A schematic view of the apparatus is shown in Figure 2A. MEH-PPV (American Dye Source), dimethylsulfoxide, and $o$-dichlorobenzene (Sigma-Aldrich) were used as received.

Ultrafast OHD-OKE Spectroscopy. We have performed ultrafast spectroscopy experiments as described previously. ${ }^{39}$ Briefly, femtosecond pulses are split into pump (95\%) and probe $(5 \%)$ beams. Pulse polarization is 90 and $45^{\circ}$ for the pump and probe, respectively. Additionally, a quarter waveplate set to $\pm 1^{\circ}$ was added in the probe beam path. We combine the Kerr transients recorded at both +1 and $-1^{\circ}$ to remove the homodyne signal component. A motorized delay stage controls the time delay between pump and probe pulses arriving at the sample. The typical time resolution of our apparatus is $70 \mathrm{fs}$, as measured by cross-correlation frequency-resolved optical gating. We employ short time windows of 4 ps because our main purpose is to investigate intermolecular vibrational dynamics rather than overdamped orientational relaxation. We employ optical heterodyning to enhance the sensitivity of signal detection using lock-in amplification with an optical chopper operating at $90 \mathrm{~Hz}$.

Langevin Dynamics Simulations. We model reaction kinetics as diffusive motion on an asymmetric double-well potential energy surface by solving the Langevin equation of motion for this potential using

$$
\gamma \frac{\mathrm{d} x}{\mathrm{~d} t}=-\frac{1}{m} \frac{\mathrm{d} V(x)}{\mathrm{d} x}+\frac{1}{m} \delta F(t)
$$

where $x$ is the particle position, $m$ is the particle mass, $\gamma$ is the friction coefficient, $\delta F(t)$ is a random force with zero mean and variance

$$
\left\langle\delta F(t) \delta F\left(t^{\prime}\right)\right\rangle=2 \gamma k_{\mathrm{B}} T \delta\left(t-t^{\prime}\right)
$$

where $k_{\mathrm{B}}$ is Boltzmann's constant. The resulting trajectories were used to build particle distribution histograms, which were then fit to a Boltzmann distribution for $x$

$$
\rho=\rho_{0} \mathrm{e}^{-\beta V(x)}
$$

From the numerically retrieved energy landscapes, rate constants are calculated using

$$
k_{\text {Kramers }}=\frac{\omega_{0} \omega_{\mathrm{b}}}{2 \pi \gamma} \mathrm{e}^{-\beta V_{0}}
$$

where $\omega_{0}$ is the reactant well frequency and $\omega_{\mathrm{b}}$ is the barrier frequency.

In addition, we calculate dwell-time distributions using

$$
D(t)=1-\frac{1}{N} \sum_{t_{i}<t} p\left(t_{i}\right)
$$

where $N$ is the total number of dwell times and $p\left(t_{i}\right)$ are the number of dwell times with length $t_{i}$.

Line shape Analysis. We consider a two-electronic-level system coupled to a general $\mathrm{MBO}$ model with arbitrary damping. ${ }^{30}$ The fluorescence line shape is

$$
\sigma_{f}(\omega)=\frac{1}{\pi} \operatorname{Re} \int_{0}^{\infty} \exp \left[\mathrm{i}\left(\omega-\omega_{\text {eg }}+2 \lambda\right) t-g^{*}(t)\right] \mathrm{d} t
$$

where $\omega_{\mathrm{eg}}$ is the energy gap frequency, $2 \lambda$ is the Stokes shift, and the complex line shape function $g(t)$ is

$$
\begin{aligned}
g(t)= & \frac{1}{2 \pi} \int_{-\infty}^{\infty} \mathrm{d} \omega \frac{\tilde{C}^{\prime \prime}(\omega)}{\omega^{2}}[1+\operatorname{coth}(\beta \hbar \omega / 2) \\
& \left.\left(\mathrm{e}^{-\mathrm{i} \omega t}+\mathrm{i} \omega t-1\right)\right]
\end{aligned}
$$

where $\beta=\left(k_{\mathrm{B}} T\right)^{-1}$. We note that $g^{*}(t)$ is the complex conjugate of line shape function $g(t)$. The Brownian oscillator Hamiltonian includes an explicit treatment of the bath oscillators and their coupling to the primary oscillators. Thus, the spectral density for the $j$ th oscillator is

$$
\tilde{C}_{j}^{\prime \prime}(\omega)=\frac{\hbar}{2 m_{j}} \frac{\omega \gamma_{j}}{\left(\omega_{j}^{2}-\omega^{2}\right)^{2}+\omega^{2} \gamma_{j}^{2}}
$$

where $\omega_{j}$ and $\gamma_{j}$ are the bath oscillator frequency and coupling, respectively. The full spectral density is then given by

$$
\tilde{C}(\omega)=\sum_{j} \frac{m_{j} \omega_{j}^{2} d_{j}}{\hbar} \tilde{C}_{j}^{\prime \prime}(\omega)
$$

where $m_{j}$ is the $j$ th nuclear mode mass and $d_{j}$ is the $j$ th mode equilibrium configuration displacement between the two electronic states.

\section{ASSOCIATED CONTENT}

\section{Supporting Information}

The Supporting Information is available free of charge on the ACS Publications website at DOI: 10.1021/acsomega.7b00004.

Optical microscopy and electrodynamics simulations characterization, results of control experiments, polymer energy landscape, and ultrafast spectroscopy fitting parameters (PDF)

\section{AUTHOR INFORMATION}

\section{Corresponding Author}

*E-mail: nome@iqm.unicamp.br.

ORCID

René A. Nome: 0000-0003-4804-3395 


\section{Present Addresses}

"School of Physics, The University of Sydney, Sydney, NSW 2006, Australia (J.G.H.).

${ }^{\S}$ Department of Biology, Centro Universitário Claretiano de Batatais, Batatais, SP 14300-000, Brazil (J.L.).

Notes

The authors declare no competing financial interest.

\section{ACKNOWLEDGMENTS}

We thank CNPq, SAE-UNICAMP, and FAPESP for undergraduate research fellowships awarded to A.F.C., J.L., and G.A.M. Financial support from CNPq, CAPES, FAPESP, INCT-Cat is gratefully acknowledged. We thank the reviewers for their comments and suggestions.

\section{REFERENCES}

(1) Nitzan, A. Chemical Dynamics in Condensed Phases-Relaxation, Transfer, and Reactions in Condensed Molecular Systems; Oxford University Press: New York, 2010; pp 483-533.

(2) Chandler, D. Introduction to Modern Statistical Mechanics; Oxford University Press: New York, 1987; pp 234-265.

(3) Holfelder, F.; Kirby, A. J. From Enzyme Models to Model Enzymes; Oxford University Press: Oxford, 2009; pp 29-41.

(4) Turro, N. J. Modern Molecular Photochemistry. University Science Books: Herndon, VA, 1991; pp 362-413.

(5) Betzig, E.; Chichester, R. J. Single molecules observed by nearfield scanning optical microscopy. Science 1993, 262, 1422-1425.

(6) Xie, X. S.; Dunn, R. C. Probing single-molecule dynamics. Science 1994, 265, 361-364.

(7) Xie, X. S. Single-molecule spectroscopy and dynamics at room temperature. Acc. Chem. Res. 1996, 29, 598-606.

(8) Nome, R. A.; Guffey, M. J.; Scherer, N. F.; Gray, S. K. Plasmonic Interactions and Optical Forces between Au Bipyramidal Nanoparticle Dimers. J. Phys. Chem. A 2009, 113, 4408-4415.

(9) Kou, S. C.; Cherayil, B. J.; Min, W.; English, B. P.; Xie, X. S. Single-molecule Michaelis-Menten equations. J. Phys. Chem. B 2005, 109, 19068-19081.

(10) Krichevsky, O.; Bonnet, G. Fluorescence Correlation Spectroscopy: the technique and its applications. Rep. Prog. Phys. 2002, 65, 251-297.

(11) Chung, H. S.; Eaton, W. A. Single-molecule fluorescence probes dynamics of barrier crossing. Nature 2013, 502, 685-688.

(12) Qu, X.; Wu, D.; Mets, L.; Scherer, N. F. Nanometer-localized multiple single-molecule fluorescence microscopy. Proc. Natl. Acad. Sci. U.S.A. 2004, 101, 11298-11303.

(13) Fields, A. P.; Cohen, A. E. Electrokinetic trapping at the one nanometer limit. Proc. Natl. Acad. Sci. U.S.A. 2011, 108, 8937-8942.

(14) Levene, M. J.; Korlach, J.; Turner, S. W.; Foquet, M.; Craighead, H. G.; Webb, W. W. Zero-mode waveguides for single-molecule analysis at high concentrations. Science 2003, 299, 682-686.

(15) Wang, S.-L.; Huang, X.-J.; Fang, Z.-L.; Dasgupta, P. K. A miniaturized liquid core waveguide-capillary electrophoresis system with flow injection sample introduction and fluorometric detection using light-emitting diodes. Anal. Chem. 2001, 73, 4545-4549.

(16) Dasgupta, P. K.; Genfa, Z.; Poruthoor, S. K.; Caldwell, S.; Dong, S.; Liu, S. Y. High-sensitivity gas sensors based on gas-permeable liquid core waveguides and long-path absorbance detection. Anal. Chem. 1998, 70, 4661-4669.

(17) Dasgupta, P. K.; Zhang, G. F.; Li, J. Z.; Boring, C. B.; Jambunathan, S.; Al-Horr, R. Luminescence detection with a liquid core waveguide. Anal. Chem. 1999, 71, 1400-1407.

(18) Dallas, T.; Dasgupta, P. K. Light at the end of the tunnel: recent analytical applications of liquid-core waveguides. TrAC, Trends Anal. Chem. 2004, 23, 385-392.

(19) Schmidt, M.; Cubillas, A. M.; Taccardi, N.; Euser, T. G.; Cremer, T.; Maier, F.; Steinruck, H. P.; Russell, P. S.; Wasserscheid, P.;
Etzold, B. J. M. Chemical and (Photo)-Catalytical Transformations in Photonic Crystal Fibers. Chem CatChem 2013, 5, 641-650.

(20) Cubillas, A. M.; Schmidt, M.; Scharrer, M.; Euser, T. G.; Etzold, B. J. M.; Taccardi, N.; Wasserscheid, P.; Russell, P. S. Ultra-low concentration monitoring of catalytic reactions in photonic crystal fiber. Chem. - Eur. J. 2012, 18, 1586-1590.

(21) Chen, J. S. Y.; Euser, T. G.; Farrer, N. J.; Sadler, P. J.; Scharrer, M.; Russell, P. S. Photochemistry in Photonic Crystal Fiber Nanoreactors. Chem. - Eur. J. 2010, 16, 5607-5612.

(22) Lande, D. R., Ed. Handbook of Chemistry and Physics; CRC, 2010.

(23) Lakowicz, J. R. Principles of Fluorescence Spectroscopy; Springer: New York, 2006; pp 757-795.

(24) Hagler, T. W.; Pakbaz, K.; Voss, K. F.; Heeger, A. J. Enhanced order and electronic delocalization in conjugated polymers oriented by gel processing in polyethylene. Phys. Rev. B: Condens. Matter Mater. Phys. 1991, 44, 8652-8666.

(25) Scurlock, R. D.; Wang, B.; Ogilby, P. R.; Sheats, J. R.; Clough, R. L. Singlet oxygen as a reactive intermediate in the photodegradation of an electroluminescent polymer. J. Am. Chem. Soc. 1995, 117, 1019410202.

(26) Cumpston, B. H.; Jensen, K. F. Photooxidative stability of substituted poly(phenylene vinylene) (PPV) and poly(phenylene acetylene) (PPA). J. Appl. Polym. Sci. 1998, 69, 2451-2458.

(27) Bianchi, R. F.; Balogh, D. T.; Tinani, M.; Faria, R. M.; Irene, E. A. Ellipsometry study of the photo-oxidation of poly[(2-methoxy-5hexyloxy)-p-phenylenevinylene]. J. Polym. Sci., Part B: Polym. Phys. 2004, 42, 1033-1041.

(28) Neugebauer, H.; Brabec, C. J.; Hummelen, J. C.; Janssen, R. A. J.; Sariciftci, N. S. Stability studies and degradation analysis of plastic solar cell materials by FTIR spectroscopy. Synth. Met. 1999, 102, $1002-1003$

(29) Brabec, C. J.; Johannson, H.; Padinger, F.; Neugebauer, H.; Hummelen, J. C.; Sariciftci, N. S. Photoinduced FT-IR spectroscopy and $\mathrm{CW}$-photocurrent measurements of conjugated polymers and fullerenes blended into a conventional polymer matrix. Sol. Energy Mater. Sol. Cells 2000, 61, 19-33.

(30) Mukamel, S. Principles of Nonlinear Optical Spectroscopy; Oxford University Press: New York, 1995; pp 227-252.

(31) Moran, A. M.; Nome, R. A.; Scherer, N. F. Field-resolved coherent Raman spectroscopy of high frequency vibrational resonances. J. Phys. Chem. A 2006, 110, 10925-10928.

(32) Prince, R. C.; Frontiera, R. R.; Potma, E. O. Stimulated Raman Scattering: From Bulk to Nano. Chem. Rev. 2016, 117, 5070-5094.

(33) Kalanoor, B. S.; Ronen, M.; Oren, Z.; Gerber, D.; Tischler, Y. R. New Method to Study the Vibrational Modes of Biomolecules in the Terahertz Range Based on a Single-Stage Raman Spectrometer. ACS Omega 2017, 2, 1232-1240.

(34) Lotshaw, W. T.; McMorrow, D.; Kalpouzos, C.; KenneyWallace, G. A. Femtosecond dynamics of the optical Kerr effect in liquid nitrobenzene and chlorobenzene. Chem. Phys. Lett. 1987, 136, 323-328.

(35) Thompson, M. A.; Lew, M. D.; Moerner, W. E. Extending Microscopic Resolution with Single-Molecule Imaging and Active Control. Annu. Rev. Biophysics 2012, 41, 321-342.

(36) Miller, E. D.; Jones, M. L.; Jankowski, E. Enhanced Computational Sampling of Perylene and Perylothiophene Packing with Rigid-Body Models. ACS Omega 2017, 2, 353-362.

(37) COMSOL Multiphysics. Home Page. http://www.comsol.com (accessed May 12, 2017).

(38) ImageJ. Home Page. http://imagej.nih.gov/ij/ (accessed May $12,2017)$.

(39) Loiola, P. T. C.; Dias, W. D.; Souza, M. S.; Ferbonink, G. F.; Zaniolo, M. G.; Cerini, M. F.; Atvars, T. D. Z.; Nome, R. A. Ethyl stearate:ethanol binary mixtures investigated by ultrafast OKE spectroscopy, optical microscopy, dynamic light scattering, and rheology. J. Phys. Org. Chem. 2014, 27, 316-321. 